\title{
Unsuspected Infection Is Infrequent in Asymptomatic Outpatients With Refractory Ascites Undergoing Therapeutic Paracentesis
}

\author{
Mark A. Jeffries, D.O., Mark A. Stern, M.D., Naresh T. Gunaratnam, M.D., and Robert J. Fontana, M.D. \\ Division of Gastroenterology, Department of Internal Medicine, University of Michigan Medical School, Ann \\ Arbor, Michigan
}

OBJECTIVE: Large-volume paracentesis is a safe and effective means of treating patients with refractory ascites. However, there is limited information regarding the need for ascitic fluid studies in asymptomatic outpatients presenting for therapeutic paracentesis. The aim of this prospective study was to define the incidence and natural history of peritoneal fluid infection in asymptomatic outpatients undergoing therapeutic paracentesis.

METHODS: Over a 13-month period, 118 therapeutic paracenteses were performed in 29 outpatients with decompensated cirrhosis (Child-Pugh class $\mathrm{B}=38 \%, \mathrm{C}=62 \%$ ). After a brief medical history and physical examination, ascitic fluid cell count with differential and culture were obtained from all participating subjects. Seven (24\%) of the subjects were receiving norfloxacin prophylaxis, accounting for antibiotic coverage during $40 \%$ of the procedures performed. The clinical course and outcome of study subjects during a mean follow-up of 137 days was reviewed.

RESULTS: All 118 (100\%) of the ascitic fluid samples demonstrated absolute neutrophil counts of $<250 / \mathrm{mm}^{3}$ (mean $=$ $6.5 \pm 22.5 \mathrm{pmn} / \mathrm{mm}^{3}$ ). Asymptomatic bacterascites was identified from three of the 118 (2.5\%) fluid samples, but all of these subjects spontaneously recovered without treatment or sequelae. During follow-up, six episodes of symptomatic or hospital-associated peritoneal fluid infection were identified in study participants, emphasizing the importance of fluid studies in other clinical settings.

CONCLUSIONS: Although further studies are needed, the routine culture of ascitic fluid in asymptomatic outpatients with refractory ascites requiring therapeutic paracentesis may not be necessary when there is a low index of suspicion for occult infection. In circumstances of clinical uncertainty, however, obtaining ascitic fluid cell counts with differential is recommended to insure patient safety. (Am J Gastroenterol 1999;94:2972-2976. (C) 1999 by Am. Coll. of Gastroenterology)

\section{INTRODUCTION}

Refractory ascites (RA), defined as fluid overload that is unresponsive to sodium restriction and high-dose diuretics, is estimated to occur in $<10 \%$ of patients with cirrhotic ascites (1). In light of the poor short-term survival in these individuals, it is currently recommended that patients with RA be considered for liver transplantation $(2,3)$. In both transplant candidates and other patients with RA, intermittent therapeutic large-volume paracentesis (LVP) is considered a safe, simple, and effective treatment strategy $(3,4)$.

Patients with advanced and severe liver disease such as those with refractory ascites appear to be at increased risk of developing peritoneal fluid infection (5). Although the outcome with spontaneous bacterial peritonitis (SBP) and SBP variants has improved over the last decade, the in-hospital and 1-yr mortality of patients with SBP remain approximately $30 \%$ and $50 \%$, respectively (6-8). At presentation, as many as one-third of patients with infected peritoneal fluid do not manifest overt signs or symptoms such as fever or abdominal pain (6). Furthermore, 7-27\% of patients with cirrhotic ascites harbor occult peritoneal fluid infection at the time of hospital admission $(6,9,10)$. Therefore, a high index of suspicion with a low threshold to perform a diagnostic paracentesis is required to make a rapid diagnosis of this potentially life-threatening infection in various clinical settings.

With an increasing emphasis on cost containment in the delivery of medical care, many patients with RA are undergoing LVP in the outpatient setting. Although many of these patients are at increased risk for SBP due to their advanced liver disease, low protein ascites, and prior episode(s) of SBP, the need for routine ascitic fluid analysis in the outpatient setting remains unclear (11). The issue is further complicated by the fact that some patients are now receiving primary or secondary antibiotic prophylaxis to prevent SBP. In our experience, there is considerable variability in clinical practice with regards to the need and type of diagnostic ascitic fluid studies to perform in the outpatient setting. Therefore, the aim of this study was to prospectively determine the incidence and natural history of peritoneal fluid 
infection in asymptomatic outpatients with RA presenting for therapeutic paracentesis.

\section{MATERIALS AND METHODS}

\section{Patient Population}

Outpatients with cirrhosis and RA presenting to the Medical Procedure Unit at the University of Michigan Medical Center for LVP were invited to participate in this study from April 1, 1997 to May 15, 1998. A brief medical history and physical exam was performed before each LVP by a gastroenterology fellow or attending physician. Inclusion criteria were age $\geq 18 \mathrm{yr}$ with diuretic refractory or intolerant (i.e., develop prerenal azotemia or electrolyte imbalance) cirrhotic ascites requiring therapeutic LVP. Exclusion criteria included symptoms of possible peritonitis such as recent fever, chills, or abdominal pain not thought to be related to fluid accumulation alone. Subjects with moderate to severe abdominal tenderness or peritoneal signs on examination were excluded. Other exclusion criteria included evidence of acute decompensation in liver disease with gastrointestinal bleeding, worsening hepatic encephalopathy, or acute renal insufficiency with a serum creatinine $>2.5 \mathrm{mg} / \mathrm{dl}$. In addition, subjects with malignant ascites and noncirrhotic ascites were excluded. Any subject receiving prescription antibiotics for a documented bacterial infection within 2 wk of LVP were excluded. Subjects receiving oral antibiotics for primary or secondary prophylaxis for SBP were included. The protocol was reviewed by the local Institutional Review Board and written informed consent was obtained from all participating subjects.

During the study period, 227 outpatient paracenteses were performed at our institution. Twenty-nine eligible subjects were enrolled at the time of 118 therapeutic paracenteses and form the basis of this report.

\section{Procedure}

Large-volume paracentesis was performed in the standard fashion. A site in the midline suprapubic or lower quadrant area was localized by percussion and prepped using sterile technique. A 14-gauge catheter was inserted into the peritoneal cavity until a free flow of ascitic fluid was obtained. The needle trocar was removed and fluid samples for diagnostic studies were obtained. The catheter was connected to a wall-mounted suction unit with the intent to drain the peritoneal cavity to the greatest extent possible, or total paracentesis. In most instances, ascitic fluid was drained within 30-90 min of needle insertion. Details of the procedure, including the number of needle passes, fluid appearance, volume removed, and complications, were prospectively recorded. Immediate periprocedural complications included hypotension, defined as a decrease in systolic blood pressure of $>20 \mathrm{~mm} \mathrm{Hg}$ or a systolic blood pressure $<90 \mathrm{~mm} \mathrm{Hg}$ upon completion of the procedure, intraperitoneal or site bleeding requiring transfusion or hospitalization, new-onset abdominal pain, or leakage at the needle- insertion site. Human serum albumin was infused per predetermined dosing guidelines at the discretion of the attending physician.

Laboratory test charges for ascitic fluid studies at the University of Michigan Hospitals were obtained from the Department of Laboratory Services. Estimates of the corresponding Medicare reimbursement for the fluid studies performed were obtained from published fee schedules for CPT codes 89051 (fluid cell count with differential), 87075 (anaerobic culture), and 87070 (aerobic culture).

\section{Ascitic Fluid Analysis}

Using sterile technique, $10 \mathrm{ml}$ of ascitic fluid was inoculated at the bedside into Bactec-T/Alert FAN aerobic and anaerobic blood culture bottles (Organon Teknika, Durham, NC) (12). Ascitic fluid cell counts with manual differential of the white blood cell count was performed on all specimens using standard laboratory techniques. Peritoneal fluid results were categorized as follows: spontaneous bacterial peritonitis $(\mathrm{SBP})=$ fluid cell count of $\geq 250$ polymorphonuclear leukocytes $(\mathrm{PMN}) / \mathrm{mm}^{3}$ with a positive culture; culturenegative neutrocytic ascites $(\mathrm{CNNA})=$ fluid cell count of $\geq 250 \mathrm{PMN} / \mathrm{mm}^{3}$ and a negative culture after 7 days; monomicrobial nonneutrocytic bacterascites $(\mathrm{MNB})=$ fluid cell count of $<250 \mathrm{PMN} / \mathrm{mm}^{3}$ and a single positive bacterial culture result; normal or uninfected $=$ fluid cell count of $<250 \mathrm{PMN} / \mathrm{mm}^{3}$ and a negative culture after 7 days. All subjects with MNB underwent repeat diagnostic paracentesis within 5 days of a positive culture report to detect progression to SBP or resolution of bacterascites.

\section{Subject Follow-Up}

The cumulative incidence of peritoneal fluid infection and the clinical outcome of study participants during the 13month study period were determined by chart review.

\section{Statistics}

Statistical analysis of the data was performed using StatView 4.5 (Abacus Concepts Inc., Berkeley, CA). Values are expressed as the mean $\pm \mathrm{SD}$.

\section{RESULTS}

Clinical characteristics of the 29 eligible subjects with refractory cirrhotic ascites undergoing 118 therapeutic paracenteses are summarized in Table 1 . All subjects entered into the protocol had a low ascitic fluid albumin and a serum-ascites albumin gradient $>1.1 \mathrm{~g} / \mathrm{dl}$ (10). The etiology of cirrhosis was related to alcohol and hepatitis $\mathrm{C}$ in the majority of subjects. All subjects had evidence of advanced liver disease with 38\% Child-Pugh class B and 62\% ChildPugh class C. In addition, $17 \%$ had a documented episode of prior SBP and 38\% were awaiting liver transplantation. Overall, 24\% of the subjects were receiving oral antibiotics for primary or secondary prevention of SBP, accounting for antibiotic coverage during $40 \%$ of the procedures performed. 
Table 1. Clinical Characteristics of Study Subjects $(\mathrm{n}=29)$

\begin{tabular}{lc}
\hline Mean age, yr & $55 \pm 11.4$ (range, 37-76 yr) \\
Gender & 23 men, 6 women \\
Etiology of cirrhosis & $12(41.4 \%)$ \\
Alcohol & $6(20.7 \%)$ \\
Alcohol and hepatitis C & $5(17.2 \%)$ \\
Hepatitis C & $2(6.9 \%)$ \\
Cryptogenic & $4(13.8 \%)$ \\
Other & \\
Child-Pugh class & $11(38 \%)$ \\
B & $18(62 \%)$ \\
C & $11(38 \%)$ \\
Transplant candidates & $7(24 \%)$ \\
Antibiotic prophylaxis &
\end{tabular}

The results from the 118 paracenteses with ascitic fluid analysis are summarized in Table 2. Therapeutic paracentesis was performed with the intent of total paracentesis whenever possible. The procedure was well tolerated by study subjects, with no serious complications identified. In all cases, the fluid cell count was $<250 \mathrm{PMN} / \mathrm{mm}^{3}$. Three of the 118 fluid specimens grew Micrococcus, Klebsiella Oxytoca, and coagulase-negative Staphylococcus species, respectively. All of the positive cultures exhibited growth within 4 days of inoculation. A careful review of the procedure notes failed to identify any technical difficulties encountered during these procedures. Two of the three positive cultures occurred in a 47-yr-old man with cryptogenic cirrhosis receiving norfloxacin $400 \mathrm{mg}$ per day for primary SBP prophylaxis. This patient underwent a total of 38 LVPs over the study period. Subject follow-up after the report of a positive culture included a repeat paracentesis with fluid studies within 5 days. In each instance, the subject remained asymptomatic and follow-up fluid studies revealed no evidence of progressive or persistent infection.

The clinical course and outcome of the 29 study subjects followed during the study period are summarized in Table 3. With a mean follow-up of $137 \pm 103$ days, seven (24\%) subjects died, six (21\%) underwent liver transplantation, 11 $(38 \%)$ were alive at their last visit, and five $(17 \%)$ were lost to follow-up. The identified causes of death included hepatorenal syndrome in three subjects and progressive liver failure in the remaining four. During follow-up, six subjects (21\%) developed discrete episodes of symptomatic peritoneal fluid infection that were diagnosed under clinical circumstances independent of a study-related paracentesis.

Table 2. Ascitic Fluid Analysis at LVP $(n=118)$

\begin{tabular}{|c|c|}
\hline Mean no. of LVP/subject & $4.1 \pm 7.5($ range, $1-36)$ \\
\hline Mean volume removed & $9.4 \pm 4.9 \mathrm{~L}$ (range, $2-20.8)$ \\
\hline No. receiving albumin & $103(87 \%)$ \\
\hline Mean albumin infused & $24.2 \pm 16.0 \mathrm{~g}$ \\
\hline Mean fluid $\mathrm{WBC} / \mathrm{mm}^{3}$ & $107 \pm 94.5 / \mathrm{mm}^{3}$ (range, $8-538 / \mathrm{mm}^{3}$ ) \\
\hline Mean fluid PMN/mm 3 & $6.5 \pm 22.5 / \mathrm{mm}^{3}\left(\right.$ range, $\left.0-204 / \mathrm{mm}^{3}\right)$ \\
\hline Positive cultures* & $3(2.5 \%)$ \\
\hline
\end{tabular}

Table 3. Ascitic Fluid Infection Identified at the Time of a Nonstudy-related Paracentesis*

\begin{tabular}{|c|c|c|}
\hline Infection $\dagger$ & Presentation & Culture Results \\
\hline CNNA & Fever, abdominal pain & No growth \\
\hline SBP & Fever, abdominal pain & Escherchia coli \\
\hline $\mathrm{MNB} \ddagger$ & Encephalopathy, N/V & Streptococcus sp. \\
\hline MNB & At admission & Serratia $s p$ \\
\hline MNB & Abdominal pain & Staphylococcus sp. \\
\hline MNB & Fever, other infection & Pseudomonas sp. \\
\hline \multicolumn{3}{|c|}{ 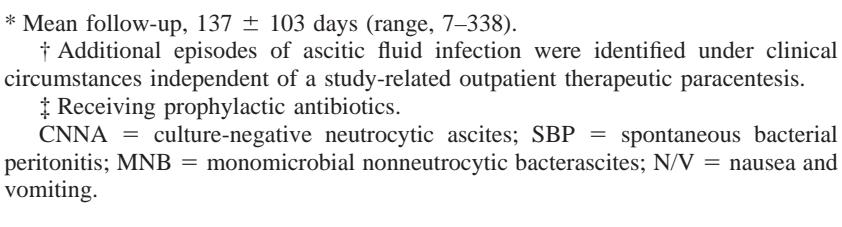 } \\
\hline
\end{tabular}

Four cases of MNB and one case each of SBP and CNNA were diagnosed immediately before or upon admission to the hospital in patients with clinical symptoms (Table 3). Only one of the six subjects was receiving prophylactic antibiotics. All of these episodes of symptomatic peritoneal fluid infection were successfully treated with antibiotics without any associated in-hospital mortality. There was no apparent temporal relationship between these episodes of infection and the performance of an outpatient therapeutic LVP.

The hospital charges and Medicare reimbursement for the ascitic fluid cell counts and culture are summarized in Table 4. Assuming that the incidence of peritoneal fluid infection is $2.5 \%$ in asymptomatic outpatients, the estimated cost to diagnose a single case of monomicrobial bacterascites in a comparable patient population would be $\$ 1320-\$ 9600$ using Medicare reimbursement and University of Michigan Medical Center institutional charges, respectively.

\section{DISCUSSION}

Patients with refractory ascites are at increased risk of developing potentially serious and life-threatening spontaneous peritoneal fluid infection, but little information regarding the need for routine ascitic fluid analysis at the time of outpatient therapeutic paracentesis is available. We set out to prospectively study ascitic fluid cell counts and cultures obtained at the time of therapeutic LVP in outpatients

Table 4. Estimated Hospital Charges and Medicare Reimbursement for Ascitic Fluid Studies

\begin{tabular}{|c|c|c|}
\hline Ascitic Fluid Test & UMMC* & Medicare $\dagger$ \\
\hline Fluid cell count with differential & $\$ 92$ & $\$$ \\
\hline Fluid culture (aerobic/anaerobic) & $\$ 148$ & 25 \\
\hline Total charge per procedure & $\$ 240$ & 33 \\
\hline $\begin{array}{l}\text { Cost per case of MNB identified } \\
=\text { Total charge per procedure/ } \\
\text { ( } 2.5 \text { case of MNB identified/ } \\
100 \text { procedures) }\end{array}$ & $\$ 9600$ & $\$ 1320$ \\
\hline
\end{tabular}


Table 5. Studies of Ascitic Fluid Analysis During Outpatient Large-Volume Paracentesis

\begin{tabular}{|c|c|c|c|}
\hline Study & $\begin{array}{c}\% \text { Patients With } \\
\text { Cell Count } \geq 250 \\
\text { PMN } / \mathrm{mm}^{3}\end{array}$ & $\begin{array}{l}\% \text { Patients With } \\
\text { Positive Cultures }\end{array}$ & $\begin{array}{l}\text { Outcome of Patients With } \\
\text { Positive Cultures }\end{array}$ \\
\hline Stern et al. $(21)^{*}$ & $0 \%$ & $0 \%(0 / 37)$ & \\
\hline Kolle et al. (22) & $0 \%$ & $2.3 \%(4 / 173)$ & No treatment or sequelae \\
\hline Jeffries et al. $\dagger$ & $0 \%$ & $2.5 \%(3 / 118)$ & No treatment or sequelae \\
\hline
\end{tabular}

with refractory ascites. We found that none of the 118 fluid specimens demonstrated elevated neutrophil counts suggestive of bacterial peritonitis. Furthermore, the bedside inoculation of blood culture bottles grew bacterial microorganisms in only three of 118 (2.5\%) samples. With fluid cell counts of $<250 \mathrm{PMN} / \mathrm{mm}^{3}$, these cases were classified as $\mathrm{MNB}$, an SBP variant with a more favorable prognosis than SBP or CNNA $(13,14)$. Close monitoring of subjects with MNB demonstrated spontaneous clearance of bacterascites in all three cases. This favorable outcome in subjects with asymptomatic MNB is consistent with prior studies that demonstrate a favorable prognosis in the absence of symptoms $(14,15)$.

The low rate of peritoneal fluid infection observed in our study may be due to several factors. Subjects with overt signs or symptoms suggestive of peritoneal fluid infection, acute decompensation in liver disease, and those being admitted to the hospital for other reasons were excluded from this protocol, as it is standard of care to perform a diagnostic paracentesis in these high-risk settings (16). Secondly, antibiotic prophylaxis with norfloxacin was used by $24 \%$ of study subjects, accounting for antibiotic coverage during $40 \%$ of the procedures performed. This may have resulted in a lower-than-expected rate of peritoneal fluid infection, as previous studies have demonstrated the efficacy of antibiotic prophylaxis in high-risk patient populations $(17,18)$.

It is unlikely that the low rate of peritoneal fluid infection observed was due to suboptimal culture methods and techniques. Ten milliliters of ascitic fluid was directly inoculated into aerobic and anaerobic blood culture bottles at the bedside using sterile technique. This culture method has been previously shown to be superior to standard culture methods in the detection of ascitic fluid pathogens $(12,19)$. Conversely, highly sensitive culture techniques may theoretically overestimate the prevalence of true peritoneal fluid infection due to growth of contaminants. The three cases of bacterascites were classified as MNB, as prior reports have identified the isolated microorganisms as potential pathogens (20). However, the clinical significance of a culture growing coagulase-negative staphylococci, a frequent skin contaminant, remains unclear.

Despite the low incidence of peritoneal fluid infection at the time of outpatient LVP, the study population represented a high-risk group. All of the study subjects had RA, $62 \%$ were Child-Pugh class C cirrhotics, and $17 \%$ had a prior history of documented SBP. During a mean follow-up of 137 days, six subjects (21\%) were diagnosed with symptomatic SBP or SBP variants under clinical circumstances independent of a study-related procedure (Table 3). Fortunately, all six cases were successfully treated with antibiotics without any associated in-hospital mortality. Nonetheless, overall mortality remained predictably high in our study population, with only $55 \%$ of subjects surviving without liver transplantation.

Only two other studies published in abstract form have addressed the issue of whether ascitic fluid should be analyzed at the time of therapeutic paracentesis in asymptomatic outpatients (Table 5). In a retrospective study of 37 outpatient LVPs performed at Emory University, ascitic fluid cell counts and cultures revealed no evidence of peritoneal fluid infection (21). Similarly, in a prospective study from Barcelona, 173 ascitic fluid samples were analyzed from 51 asymptomatic stable cirrhotics with RA (22). All ascitic fluid cell counts were $<250 \mathrm{PMN} / \mathrm{mm}^{3}$ and only four cultures $(2.3 \%)$ grew bacterial microorganisms. These infections, classified as asymptomatic MNB, reportedly cleared without treatment or sequelae. The incidence of MNB and the favorable clinical course of infected patients was remarkably similar to that observed in the present study.

The low incidence of peritoneal fluid infection reported in these studies should not overshadow the importance of diagnostic fluid studies in the inpatient or ambulatory outpatient setting under other circumstances. For example, patients with cirrhosis and new-onset ascites, acute decompensation of liver disease, or signs and symptoms of peritonitis should undergo expedient evaluation of ascitic fluid to exclude potentially serious and life-threatening infection. In clinical practice, patients requiring therapeutic LVP may present with less ominous but concerning symptoms of vague abdominal discomfort, mild tenderness on exam, or subtle changes in affect that may be suggestive of occult infection. It would be reasonable in such instances of clinical uncertainty to obtain ascitic fluid cell count with differential. This approach would rapidly screen for SBP and CNNA, which require immediate intervention without the additional expense of cultures. However, if the ascitic fluid were cloudy at the time of therapeutic paracentesis, it would be prudent to obtain not only cell counts but a bacterial culture as well.

Our study results demonstrate that unsuspected ascitic fluid infection in asymptomatic outpatients with refractory 
ascites is uncommon (2-3\%). Because the outcome of patients with asymptomatic MNB was favorable and comparable to that reported in the literature, minimal risk would be incurred if ascitic fluid cultures were not routinely performed. Furthermore, with estimated charges of $\$ 240$ per procedure for diagnostic fluid studies (Medicare reimbursement of \$32), it would cost $\sim \$ 9600$ (Medicare reimbursement of $\$ 1320$ ) to diagnose each case of asymptomatic MNB in a comparable patient population (Table 4). Although further studies are needed, the routine culture of ascitic fluid in asymptomatic outpatients frequently receiving prophylactic antibiotics may not be necessary when there is a low index of suspicion for occult infection. In circumstances of clinical uncertainty, however, obtaining an ascitic fluid cell count with differential is recommended to insure patient safety.

\section{ACKNOWLEDGMENT}

We thank Drs. Timothy Nostrant, Grace Elta, William Chey, Jeff Barnett, William Hasler, and James Scheiman, and the Gastroenterology fellows at the University of Michigan, for their assistance in patient recruitment and enrollment. We also thank the nursing and clerical staff in the Medical Procedures Unit for their assistance.

Reprint requests and correspondence: Robert J. Fontana, M.D., Division of Gastroenterology, 3912 Taubman Center, The University of Michigan Medical Center, Ann Arbor, Michigan 481090362.

Received Apr. 16, 1999; accepted Apr. 26, 1999.

\section{REFERENCES}

1. Arroyo V, Gines P, Gerbes AL, et al. Definition and diagnostic criteria of refractory ascites and hepatorenal syndrome in cirrhosis. Hepatology 1996;23:164-76.

2. Gines P, Arroyo V, Vargas V, et al. Paracentesis with intravenous infusion of albumin as compared with peritoneovenous shunting in cirrhosis with refractory ascites. N Engl J Med 1991;325:829-35.

3. Runyon BA. Management of adult patients with ascites caused by cirrhosis. Hepatology 1998;27:264-72.

4. Runyon BA. Paracentesis of ascitic fluid: A safe procedure. Arch Intern Med 1986;146:2259-61.

5. Hoefs JC, Canawatti HN, Sapico FL, et al. Spontaneous bacterial peritonitis. Hepatology 1982;2:399-407.

6. Pinzello G, Simonetti RG, Craxi A, et al. Spontaneous bacte- rial peritonitis: A prospective investigation in predominantly nonalcoholic cirrhotic patients. Hepatology 1983;3:545-9.

7. Bac DJ. Spontaneous bacterial peritonitis: An indication for liver transplantation? Scand J Gastroenterol 1996;218S:3842.

8. Altman C, Grange JD, Amiot X, et al. Survival after a first episode of spontaneous bacterial peritonitis: Prognosis of potential candidates for orthotopic liver transplantation. J Gastroenterol Hepatol 1995;10:47-50.

9. Almdal TP, Skinhoj P. Spontaneous bacterial peritonitis in cirrhosis: Incidence, diagnosis, and prognosis. Scand J Gastroenterol 1987;22:295-300.

10. Hurwich DB, Lindor KD, Hay JE, et al. Prevalence of peritonitis and the ascitic fluid protein concentration among chronic liver disease patients. Am J Gastroenterol 1993;88: 1254-7.

11. Runyon BA. Low-protein-concentration ascitic fluid is predisposed to spontaneous bacterial peritonitis. Gastroenterology 1989;91:1343-6.

12. Ortiz J, Soriano G, Coll P, et al. Early microbiologic diagnosis of spontaneous bacterial peritonitis with BacT-ALERT. J Hepatology 1997;26:839-44.

13. Runyon BA, Hoefs JC. Culture-negative neutrocytic ascites: A variant of spontaneous bacterial peritonitis. Hepatology 1984; 4:1209-11.

14. Runyon BA. Monomicrobial nonneutrocytic bacterascites: A variant of spontaneous bacterial peritonitis. Hepatology 1990; 12:710-5.

15. Chu C, Chang K, Liaw Y. Prevalence and prognostic significance of bacterascites in cirrhotics with ascites. Dig Dis Sci 1995;40:561-5.

16. Guarner C, Soriano G. Spontaneous bacterial peritonitis. Semin Liver Dis 1997;17:203-17.

17. Gines P, Rimola A, Planas R, et al. Norfloxacin prevents spontaneous bacterial peritonitis recurrence in cirrhosis: Results of a double-blind, placebo-controlled trial. Hepatology 1990;12:716-24.

18. Soriano G, Teixedo M, Guarner C, et al. Selective intestinal decontamination prevents spontaneous bacterial peritonitis. Gastroenterology 1991;100:477-81.

19. Runyon BA, Umland ET, Merlin T. Inoculation of blood culture bottles with ascitic fluid: Improved detection of spontaneous bacterial peritonitis. Arch Intern Med 1987;147:73-5.

20. Boixeda D, DeLuis DA, Aller R, et al. Spontaneous bacterial peritonitis: Clinical and microbiologic study of 233 episodes. J Clin Gastroenterol 1996;23:275-9.

21. Stern MA, Chalasani N, Strauss RM. Is it cost effective or necessary to routinely analyze ascitic fluid in an asymptomatic outpatient population of cirrhotics? Hepatology 1994;19: 1271A (abstract).

22. Kolle L, Ortiz J, Ricart E, et al. Ascitic fluid culture is not necessary in asymptomatic cirrhotic outpatients undergoing repeated therapeutic paracentesis. Hepatology 1996;24:445A (abstract). 